\title{
Central nervous system haemangioblastoma: a clinical and genetic study of 52 cases
}

\author{
A M Boughey, N A Fletcher, A E Harding
}

\begin{abstract}
Fifty two cases of haemangioblastoma were reviewed for their clinical, genetic and prognostic features. Of 34 patients with apparently isolated cerebellar lesions, postoperative outcome was good in $79 \%$. Six isolated spinal lesions presented more insidiously and morbidity was related to incomplete resection. Twelve $(23 \%)$ of the patients definitely had von Hippel-Lindau disease (VHLD). The true proportion may be higher as this diagnosis was not definitely excluded in many of the remainder; only ten patients with seemingly isolated cerebellar tumours were appropriately investigated and two had evidence of VHLD. Four out of 26 cases (15\%) with apparently completely resected, isolated, cerebellar lesions later developed recurrent tumours. Brainstem and supratentorial haemangioblastomas were rare and were always associated with VHLD. The cerebellar or spinal haemangioblastomas due to VHLD had no distinctive clinical features compared with isolated tumours and there was considerable overlap in age of onset between the two groups of cases. All patients with an apparently isolated CNS haemangioblastoma should be investigated for evidence of von HippelLindau disease.
\end{abstract}

Haemangioblastomas may arise in isolation or as a manifestation of von Hippel-Lindau disease (VHLD); they are often multiple in the latter. Other features of VHLD include retinal angiomas, renal cell carcinoma and adenoma, phaeochromocytoma, pancreatic, renal and hepatic cysts, and epidydimal cysts and tumours. ${ }^{1}$ The peak age of presentation of haemangioblastoma is between 30 and 60 years. ${ }^{2}$ They are situated most commonly in the cerebellum but may arise also in the spinal cord or medulla oblongata; supratentorial lesions are uncommon. ${ }^{3}$ The aetiology of the tumour is unknown but interestingly the VHLD gene has been mapped to the short arm of chromosome $3^{4}$ and cytogenetically visible deletions of this chromosome have been observed in renal carcinomas. ${ }^{5}$

A question of considerable clinical importance arises when a haemangioblastoma is discovered in the absence of a family history or lesion elsewhere; this is whether or not the patient has VHLD. The proportion of cases due to VHLD is unclear; figures from 10 $40^{\circ}{ }_{0}^{67}$ have been reported. Diagnosis of VHLD is of considerable importance for two reasons. Firstly there may be other surgically treatable lesions such as renal carcinoma and phaeochromocytoma, or retinal angiomas which need early photocoagulation to avoid loss of vision. Second, if the patient has VHLD there are obvious consequences for relatives who will require genetic counselling and screening for occult lesions. Proposals for screening haemangioblastoma patients and their relatives for $\mathrm{VHLD}^{78}$ have suggested that this approach is only required if the patient is under 50 years old, on the assumption that nearly all cases of VHLD will have presented by this age. ${ }^{7}$ We present a clinical and genetic study of fifty two cases of haemangioblastoma which specifically addresses the issue of screening for VHLD.

\section{Patients and methods}

The case records of the National Hospitals for Nervous Diseases and the diagnostic index of the Department of Neuropathology were searched for all cases of haemangioblastoma seen between 1973-88. VHLD was diagnosed where there was definite evidence of a second haemangioblastoma, one or more of the other features (such as retinal angioma, renal carcinoma or phaeochromocytoma) of the syndrome, or a single haemangioblastoma and a family history of any of the features of VHLD. ${ }^{9}$ The results of the following investigations were sought in all cases: direct and indirect ophthalmoscopy, retinal fluorescein angiography, urinalysis for microscopic haematuria, 24 hour urinary vanillylmandelic acid (VMA) excretion, abdominal CT or ultrasound scanning and intravenous urography (IVU).

We contacted as many patients as possible to establish current information concerning residual symptoms and disability, and a detailed family history was taken. Of the 52 index cases, 28 were visited at home or interviewed by telephone. Five of the remaining patients lived abroad, seven had died, four refused to be seen and eight could not be traced. Adequate clinical data were obtained from the hospital notes for all cases and pedigree data for 45. Outcome was graded as follows: asymptomatic and returned to previous employment $=1 ;$ minor symptoms and returned to previous employment $=2$; significant symptoms, not able to work, independent for activities of daily living $(\mathrm{ADL})=3$; dependent on others for $\mathrm{ADL}=4$; death $=5$. 
In patients with an isolated haemangioblastoma and no family history, paternal ages were compared with expected mean values for the general population in the same years of birth, as described by Fletcher et al. ${ }^{10}$

\section{Results}

Of the 52 patients, $33(63 \%)$ were male and 19 $(37 \%$ ) female (chi-square $=3.77, \mathrm{p}>0.05$ ). Forty $(76.9 \%)$ presented with a cerebellar haemangioblastoma; in the remainder, the tumour was spinal in nine $(17 \%)$, supratentorial in two $(3.8 \%)$ and originating from the brainstem in one. Twelve $(23 \%)$ of the cases had VHLD (95\% confidence interval = $11 \cdot 6-34 \cdot 5 \%$ ).

\section{Isolated cerebellar haemangioblastomas}

There were 34 patients who presented with a cerebellar haemangioblastoma and no overt evidence of von Hippel-Lindau disease. Median age of presentation was 49.5 years (range 18-74; fig) and median duration of symptoms before presentation was four months (range 1-30). The ages of presentation for men (25-74 years, median 52.5) and women (18-70 years, median 42) did not differ significantly (Wilcoxon test: $p>0.05$ ).

Headache was the most frequent presenting symptom, occurring in $85 \%$, but in no case did it occur alone. Ataxia and nausea/vomiting or both were present in all but four patients; two of these were also confused, one had vertigo and the other diplopia. Ataxia was the most common physical sign, seen in $82 \%$, but only occurred in isolation in two cases. Papilloedema or nystagmus were also present in two thirds of these cases.

In six cases, the presenting symptoms were mild and the physical signs subtle. Vague complaints of headache, disequilibrium or mild unsteadiness and clumsiness were reported. Ataxia was minimal, with only mild impairment of tandem walking. Two of these cases had equivocal disc swelling. One patient had no signs apart from slight drowsiness.

All 34 patients had exploration of the posterior fossa and 27 initially had a macroscopically total resection of the tumour; in another six this was incomplete and one had

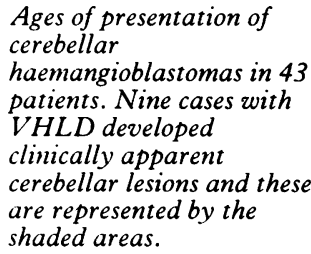



biopsy only. A further eight similar operations were subsequently performed on seven patients, seven for recurrent tumours and one complete resection after an initial biopsy. Twenty eight patients were followed up for a median period of four years (range $0 \cdot 25-22$ years); the remainder were not followed up due to postoperative death or transfer to another hospital in the United Kingdom or abroad.

Three deaths occurred in the postoperative period, each due to brainstem failure after a first operation giving a mortality rate for first operations of $8 \cdot 8 \%$. Duration of symptoms, evidence of raised intracranial pressure or of brainstem involvement were not correlated with a fatal outcome. However, three of the six patients with objective drowsiness or confusion died (exact test compared to those without: $p=$ 0.007 ), as did two of the three cases aged over 70 (exact test: $p=0.03$ ).

Six patients had seven subsequent operations for tumour recurrences; in four the initial resection had been macroscopically complete. Symptoms reappeared one to 18 years after the first lesion with a median of 12 years. There were no features indicating a high risk of clinical recurrence, including apparently incomplete first resection of a previous tumour.

A good outcome (grades 1 and 2) occurred in 27 patients $(79 \%)$ and a poor outcome (grades $3-5)$ in seven $(21 \%)$. In addition to the three patients who died, three were unable to work due to disability and one was totally dependent for ADL. Those with a poor outcome were older at presentation than those with good outcome (Wilcoxon test: $p<0.01$ ). No other factor was associated with long term disability.

\section{Isolated spinal haemangioblastomas}

There were six male patients who presented with spinal haemangioblastomas. The median age at presentation was 33 years (range 29-66) and three of the patients had noticed symptoms for over two years. The clinical presentation of these tumours was variable. Sensory symptoms (hemisensory loss or involving the upper limbs) were the most common and were present in all six cases. Urinary frequency or incontinence occurred in two. Three patients had local spinal pain, in one instance for over 10 years; in each case this was a reliable indicator of the level of the tumour. Three patients had radicular pain, referred into an arm in one and into both legs in two. Two had focal weakness involving an arm, leg or one or both hands. Marked pyramidal signs were not observed at presentation; mild increase in tone or hyperreflexia with little weakness was seen in five cases and only one had extensor plantar responses. Loss of proprioception in one arm occurred in one patient and dissociated sensory loss was seen in two with intramedullary lesions or associated syringomyelia. Radicular signs were present in half of the cases with various combinations of wasting, weakness, sensory loss and areflexia.

Three patients had laminectomy and complete excision of the haemangioblastoma. Two patients had a laminectomy and biopsy; one later had a total resection and the other received a course of radiotherapy but died 11 years later. 
Table Clinical features of 12 cases of von Hippel-Lindau disease

\begin{tabular}{|c|c|c|c|c|c|c|c|c|c|}
\hline \multirow[b]{2}{*}{ Case } & \multirow[b]{2}{*}{$F H$} & \multirow[b]{2}{*}{ Pres } & \multicolumn{4}{|c|}{ Number of tumours } & \multirow[b]{2}{*}{$\operatorname{Ret}$} & \multirow[b]{2}{*}{ Renal } & \multirow[b]{2}{*}{ Panc } \\
\hline & & & $C H B$ & $S H B$ & $S T H B$ & $B S H B$ & & & \\
\hline 1 & + & ST, R & 4 & 6 & 1 & 0 & + & - & - \\
\hline 2 & + & & 0 & 1 & 0 & 2 & - & - & - \\
\hline 3 & + & C & 11 & 1 & 0 & 1 & + & - & - \\
\hline 4 & - & $\mathbf{R}$ & 2 & 3 & 0 & 1 & + & - & - \\
\hline 5 & - & C & 1 & 0 & 0 & 0 & - & $\mathbf{M}$ & - \\
\hline 6 & - & ST & 7 & 0 & 1 & 1 & + & - & - \\
\hline 7 & + & S & 1 & 2 & 0 & 0 & + & - & - \\
\hline 8 & + & C & i & 0 & 0 & 0 & - & C & - \\
\hline 9 & + & RC & 0 & 1 & 0 & 0 & - & $\mathrm{M}, \mathrm{C}$ & - \\
\hline 10 & - & C & $>1$ & 0 & 0 & 0 & - & - & - \\
\hline 11 & + & C & 2 & 1 & 0 & 2 & - & C & C \\
\hline 12 & - & $\mathrm{C}$ & 3 & 4 & 0 & 1 & - & - & - \\
\hline
\end{tabular}

FH = family history $(+=$ present $) ;$ Pres $=$ presentation $(C=$ cerebellar, $S=$ spinal, $B=$ brainstem, $S T=$ supratentorial haemangioblastoma, $R=$ retinal angiomas, $R C=$ renal carcinoma); $C H B=$ cerebellar haemangioblastoma, SHB = spina haemangioblastoma, STHB = supratentorial haemangioblastoma, BSHB = brainstem haemangioblastoma; Ret $=$ retinal angiomas; Renal $=$ renal lesions, Panc $=$ pancreatic lesions $(M=$ malignancy, $C=$ cysts $)$.

One patient refused surgery and is now tetraplegic. In the cases where the tumour was totally resected, all had a good outcome graded 1 or 2.

\section{Cases of VHLD}

There were 12 cases of VHLD and their clinical features are shown in the table. Five of these patients were potentially at risk for VHLD at the time of presentation with a haemangioblastoma: two were known to have relatives who were affected; two had already needed treatment for retinal angiomas; another had had a nephrectomy for renal carcinoma and also had relatives who were affected.

Out of the 12 cases, the diagnosis of VHLD was initially based on the family history in four, of which two also had retinal lesions and one a previous renal carcinoma. Three others had no family history but retinal lesions were detected on fundoscopy. In the remaining five cases the diagnosis was made only after investigations revealed multiple haemangioblastomas (three cases) or visceral lesions in the kidneys or pancreas (two cases), but two of these patients (cases 8 and 11 in the table) were also later discovered to have relatives who were affected, one during the course of this study. All but one of those with positive family histories had relatives with haemangioblastomas, one of whom also had retinal angiomas; the mother of the remaining case died at the age of 36 from cerebral haemorrhage. This last patient's daughter was found to have retinal angiomas on screening.

In seven of the patients, the initial manifestation of VHLD was a cerebellar haemangioblastoma; three presented with spinal tumours, one with retinal angiomas and one with a renal carcinoma. Cerebellar haemangioblastomas were present in ten VHLD patients, were clinically apparent in nine and had no distinctive clinical features; the ages of presentation of these tumours were not significantly different from those of the non-VHLD cases (fig; Wilcoxon test: $p>0 \cdot 10$ ). Spinal haemangioblastomas were detected in eight patients and were symptomatic in six. The clinical features were similar to the isolated cases although one patient reported only transient difficulty with micturition with numbness below the waist four years before presenting with a cerebellar haemangioblastoma. In four cases there were multiple spinal lesions.

All brainstem haemangioblastomas were observed in cases of VHLD. Five patients had brainstem lesions of doubtful clinical significance, discovered by angiography or CT during evaluation of cerebellar or spinal cord tumours, which were not resected. One patient presented at the age of 21 years with clinical and radiological evidence of an extensive extrinsic medullary and cervical cord lesion after retinal angiomas had been diagnosed at the age of thirteen years. Subsequently the patient has had three craniotomies for cerebellar tumours and two operations for resection of spinal lesions. Two patients initially developed supratentorial haemangioblastomas. One presented at the age of 20 with blurred vision and headache; on examination there was papilloedema and bilateral retinal angiomas. Angiography revealed a vascular tumour in the right temporal lobe and this was resected via a temporal craniotomy. The patient developed a cerebellar haemangioblastoma three years later, and a haemangioma of the common bile duct at the age of 27 years, which recurred a year later. Another, who presented at the age of 14 years with headache, had retinal angiomas and a supratentorial haemangioblastoma. Two years later he developed raised intracranial pressure and had drainage of a cerebellar cyst and insertion of a ventriculo-atrial shunt; the supratentorial lesion was later removed leaving a residual hemiparesis and hemianopia. A symptomatic cerebellar haemangioblastoma was resected 10 years later. After a further year he had increased difficulty in walking and was found to have six spinal haemangioblastomas. $\mathrm{He}$ received a course of radiotherapy but died after developing a shunt infection and ventriculitis.

Three VHLD patients had a single haemangioblastoma. In two, this developed in the cerebellum, one with a renal carcinoma and the other with renal cysts and a positive family history; the third presented with a spinal lesion in association with renal cysts and a positive family history 11 years after a nephrectomy for a renal carcinoma.

The interval after the initial presentation before the appearance of a second VHLD lesion was between 34 months and 11 years 
(median four years). Seven VHLD patients required a total of 22 operations to remove haemangioblastomas; 13 of the 15 subsequent operations were almost certainly for additional haemangioblastomas rather than true recurrences and 10 were for cerebellar tumours.

The long term outcome among VHLD cases was not as favourable as among those with isolated haemangioblastomas. Of the twelve patients, three have died and another three are unable to work due to residual disability; half of the VHLD cases therefore have had a poor (grade 3-5) outcome. Three cases are symptomatic but at work (grade 2) and three are asymptomatic (grade 1).

Genetic studies of presumed non-VHLD cases There were 40 patients who did not meet the diagnostic criteria for VHLD. There were only 16 who had had both parents surviving to the age of 65 . The remainder had at least one parent younger than 65 or who had died before this age. Two of the 10 parents dying before 65 had strokes in the fifth decade but no other details were available. Mean paternal age at the time of the patient's birth (known for 19) was 31.79 years; this was not significantly different from the estimated population mean of 29.36 years.

Few cases were sufficiently investigated to exclude VHLD. Thirty two did not have an IVU, abdominal ultrasound or abdominal CT scan, 29 were not examined by indirect ophthalmoscopy and 13 did not have their urine examined for microscopic haematuria. Initially, there were 38 patients who presented with seemingly isolated haemangioblastomas and ten were investigated with IVU or abdominal CT scan; two of these were consequently diagnosed as having VHLD after the detection of renal cysts or carcinoma. Twenty eight patients apparently had a complete resection of a single cerebellar haemangioblastoma; two died and four out of the remaining 26 $(15 \%)$ presented later with recurrent, possibly second tumours.

\section{Discussion}

This study confirms that apparently isolated cerebellar haemangioblastoma is a more common clinical problem than VHLD or isolated spinal tumours. As previously reported, ${ }^{211}$ there was an excess of male cases, particularly for spinal lesions. For cerebellar haemangioblastomas, the median age of onset was 49.5 years which is later than in some earlier reports 6911 owing to rather more cases in this series $\left(26 \cdot 5^{\circ}{ }_{0}\right)$ over the age of 60 ; two cases $\left(6^{\circ}{ }_{10}\right)$ presented over the age of 70 . We did not confirm an earlier suggestion ${ }^{2}$ that women present earlier. Although a posterior fossa mass could be diagnosed easily in most patients, a few presented with vague symptoms and minimal physical signs. The long term results of surgical treatment for isolated cerebellar haemangioblastomas were relatively good, with a mortality of first procedures of $8.8 \%$ in contrast to figures of between $10-20^{\circ} \%$ quoted in earlier series. ${ }^{691112}$
It is difficult to be precise about the recurrence rate as some VHLD patients were known to have more than one haemangioblastoma at the time of diagnosis and nearly all subsequent operations among these cases were for additional tumours rather than recurrence of the first lesion. Among the non-VHLD patients, four developed recurrent tumours after an apparently complete resection of the first and these are likely to have developed second tumours. ${ }^{13}$ It should be noted that if an initial angiogram or the clinical features of a second presentation indicate a separate tumour, the patient has VHLD. ${ }^{9}$ Of interest is the length of time which elapsed in some cases before the second presentation, with a median of four (range 2-11) years.

For the spinal lesions there was no clinical difference in those found to have VHLD as opposed to those with isolated lesions, including the age of presentation. These tumours had a much slower evolution of symptoms than cerebellar haemangioblastomas, tending to reflect a mixture of cord and radicular involvement. Patients made a good functional recovery if the haemangioblastoma could be completely resected. It appears from our data that brainstem or supratentorial lesions occur only in VHLD; patients with such lesions should therefore be intensively investigated for other evidence of this disease.

The incidence of VHLD among all cases was $23 \%$ which may be an underestimate because few patients were fully investigated for asymptomatic lesions. In addition, many of those with an isolated haemangioblastoma cannot confidently be said to have a negative family history. In the majority of cases one or both parents had died before, or had not yet reached, the age of 65 (by which all VHLD cases in this series had presented) and were therefore still at risk of developing VHLD lesions. Some VHLD patients present after the age of 50 and others are diagnosed only at necropsy. ${ }^{14}$ Furthermore, over half of our cases have been followed up for less than five years while those with VHLD developed further manifestations up to 11 years after the first presentation (median four years). It is therefore possible that some of the isolated haemangioblastoma cases had VHLD, particularly the $15 \%$ who had recurrent cerebellar tumours after a complete initial resection. Overall, 29 of the 40 with seemingly isolated cerebellar or spinal haemangioblastomas either had recurrent tumours after apparently complete resection of the first, had been followed for less than five years or did not have a definitely negative family history. These patients are possible examples of VHLD.

We investigated the possibility that some cases with isolated haemangioblastoma and no affected relatives are due to new mutation at the VHLD locus, as appeared to be the case in five of the 12 with proven VHLD. We did not have enough pedigree information to estimate biological fitness for VHLD cases which would be a guide to the proportion due to mutation. Advanced paternal age is associated with fresh dominant mutation; $;^{15}$ this was not significantly 
increased in this study but this does not exclude the presence of cases representing new mutations.

The patient with a single haemangioblastoma and no conclusive family history or physical findings to indicate VHLD presents a considerable dilemma. A decision needs to be made as to whether or not to investigate the patient, and possibly close relatives, for subclinical lesions such as renal carcinoma, phaeochromocytoma or retinal angiomas. The absence of retinal lesions cannot be assumed to exclude VHLD because they are absent in approximately half of cases $^{1}$ and may be difficult to see on direct ophthalmoscopy if situated in the periphery of the retina. ${ }^{9}$ Also, in one report of nine VHLD families, $16 \%$ of affected individuals only had a single cerebellar haemangioblastoma. ${ }^{1}$ It has been suggested ${ }^{7}$ that cerebellar haemangioblastomas present earlier in VHLD and that the age of the patient may be a useful diagnostic guide. Moreover, screening for VHLD lesions has been proposed only for patients presenting under the age of 50 on the grounds that retinal angiomas or cerebellar haemangioblastomas present before this in VHLD. ${ }^{714}$ There are a number of exceptions to this rule in the literature; eight out of 75 patients with cerebellar haemangioblastomas reviewed by Huson et al developed symptoms after the age of 50 . It should also be noted that among cases examined at necropsy, considerable numbers of unsuspected cerebellar haemangioblastomas have been discovered. ${ }^{17}$ It appears that many of these tumours, perhaps up to $66 \%$, are never apparent in life and that in some members of VHLD pedigrees the diagnosis is only made at necropsy. ${ }^{1}$ The assumption therefore that the majority of haemangioblastomas in VHLD will present before 50 is questionable.

Of the 44 cases in this series who developed symptomatic cerebellar haemangioblastomas, ten had VHLD. We applied non-parametric statistical analysis to our age of onset data as these are not normally distributed; there appeared to be no difference between VHLD and non-VHLD cases. Two VHLD cases presented over the age of 60 ; neither of these had a family history or retinal lesions to suggest the diagnosis at presentation but renal lesions were detected on investigation and one was later found to have relatives that were affected.

In view of these observations, and the findings in this series that some cerebellar haemangioblastoma cases had recurrences which may well be second tumours, and that two of the ten investigated appropriately had silent visceral lesions, we suggest that all patients presenting with a seemingly isolated haemangioblastoma should be investigated for the presence of other features of VHLD. This should include abdominal CT, indirect ophthalmoscopy and urinary VMA estimation. This assessment should be repeated at about three yearly intervals for at least 15 years or until the age of 65, together with CT of the posterior fossa to exclude recurrent or second tumours. Clinical neurological assessment alone will not detect lesions which could give rise to fatal subarachnoid haemorrhage.

Increasing availability of magnetic resonance imaging will make noninvasive screening of both head and abdomen easier in the next few years. If a patient develops a second lesion or a relative becomes affected, then the relatives should also be screened, as recommended by Huson $e t a l$ until at least the age of 65 years. It is particularly important to identify retinal lesions, as early treatment prevents blindness. It has been proposed that screening of relatives for CNS and abdominal lesions could also cease at the age of $50 ;{ }^{8}$ in view of our results and the conclusions of an earlier study, ${ }^{1}$ this may be unwise.

We thank the physicians and surgeons at the National Hospita for Nervous Diseases for allowing us to study their patients Miss M Ellison and Miss C Kurucz for technical assistance and the Brain Research Trust for financial support.

1 Horton WA, Wong V, Eldridge R. Von Hippel-Lindau disease. Clinical and pathological manifestations in nine families with 50 affected members. Arch Intern Med 1976;136:769-77.

2 Olivecrona $\mathrm{H}$. The cerebellar angioreticulomas. J Neurosurg 1952;9:317-30.

3 Russell DS, Rubinstein LJ. Pathology of tumours of the nervous system, 4th ed. London: Arnold, 1977:116-27.

4 Seizinger BR, Rouleau GA, Ozelius LJ, et al. Von HippelLindau disease maps to the region of chromosome 3 Lindau disease maps to the region of chromosome 3
associated with renal carcinoma. Nature 1988;332:268-9.

$5 \mathrm{Zbar} B$, Branch $\mathrm{H}$, Talmadge $\mathrm{C}$, Linehan M. Loss of alleles of loci on the short arm of chromosome 3 in renal cell carcinoma. Nature 1987;327:721-4.

6 Jeffreys R. Clinical and surgical aspects of posterior fossa haemangioblastomata. J Neurol Neurosurg Psychiatry 1975;38:105-11.

7 Huson SM, Harper PS, Hourihan MD, Cole G, Weeks RD, Compston DAS. Cerebellar haemangioblastoma and von Hippel-Lindau disease. Brain 1986;109:1297-310.

8 Jennings AM, Smith C, Cole DR, et al. Von Hippel-Lindau disease in a large British family: clinicopathological features and recommendations for screening and follow up. $Q J$ Med 1988;66:233-49.

9 Melmon KL, Rosen SW. Lindau's disease. Review of the literature and study of a large kindred. $A m \mathrm{~J} \mathrm{Med}$ literature and study

10 Fletcher NA, Harding AE, Marsden CD. A genetic study of idiopathic torsion dystonia in the UK. Brain 1990;113:379-95.

11 Silver ML, Hennigar G. Cerebellar haemangioma (haemangioblastoma). A clinicopathological review of 40 cases. J Neurosurg 1952;9:484-94.

12 Palmer JJ. Haemangioblastomas. A review of 81 cases. Acta Neurochirurgica 1972;27:125-48.

13 Cramer F, Kimsey WH. The cerebellar haemangioblastomas. Review of fifty-three cases with special reference to cerebellar cysts and the association of polycythemia. Arch Neurol Psychiatry 1952;67:237-52.

14 Go RCP, Lamiell JM, Hsia YE, Yuen JMW, Paik Y. Segregation and linkage analyses of von Hippel-Lindau disease among 220 descendants from one kindred. American Journal of Human Genetics 1984;36:131-42.

15 Penrose LS. Parental age and mutation. Lancet 1955;ii:312. 